\title{
CONSERVACIÓN Y USO DE LOS RECURSOS GENÉTICOS DE ANNONACEAE EN MÉXICO
}

\author{
JORGE ANDRÉS AGUSTÍN², SERGIO D. SEGURA LEDESMA²
}

RESUMEN - Se presenta un análisis sobre la conservación y el uso de los recursos genéticos de especies frutales de la familia Annonaceae en México. El diagnóstico realizado en 2010 por la Red de Anonáceas (REMA) del Sistema Nacional de Recursos Fitogenéticos (SINAREFI) sirvió para tal fin y fue complementado por estudios recientes. Partimos de una revisión de registros de herbarios de la Red Mundial de Información sobre Biodiversidad (REMIB) además de recoger información con productores de comunidades rurales y recorridos de campo en estados del país donde las anonáceas se distribuyen. En México se encuentran 14 géneros y 63 especies de Annonaceae distribuidas principalmente en regiones tropicales del Sureste de México. La conservación in situ es esporádica, se está dando en huertos de traspatio para la guanábana (Annona muricata), chirimoya (A. cherimola), chincuya (A. purpurea) y saramuyo (A. squamosa) principalmente. La anona (A. reticulata), ilama (A. diversifolia) y chincuya (A. purpurea) son fomentadas pero no multiplicadas. La conservación ex situ se mantiene en bancos de germoplasma in vivo o colecciones de trabajo para guanábana (30 colectas), chirimoya (70 colectas) e ilama (100 colectas) solamente. Las semillas de estas especies no son ortodoxas y su conservación a mediano y largo plazo no está resuelta en México. El potencial de cultivo para este grupo de frutales es alto pero problemas de falta de variedades, fitosanidad, falta de tecnología y mercados reducidos limitan el cultivo comercial.

Termos para indexacion: Entobotânica, usos, conservación, Annonaceae.

\section{CONSERVATION AND USE OF GENETIC RESOURCES OF ANNONACEAE IN MEXICO}

\begin{abstract}
The conservation and use of genetic resources of Annonaceae of edible fruits are analyzed from a survey dated on 2010 by the National Network of Annonaceae (REMA) of the National Plant Genetic Resources (SINAREFI) and complemented by recent studies. It started with a review of herbarium records in the Global Biodiversity Information (REMIB) in addition to collecting information with producers and rural communities in field trips along the states where Annonaceae are distributed. There are in Mexico 14 genera and 63 species of Annonaceae distributed mainly in tropical regions of Southeast Mexico. In situ conservation is sporadic and occurring in backyard gardens for soursop (Annona muricata), custard apple ( $A$. cherimola) chincuya (A. purpurea) and sugar apple (A. squamosa) mainly. The anona (A. reticulata), llama (A. diversifolia) and chincuya (A. purpurea) are used but not cultivated. Ex situ conservation is maintained in vivo in gene banks or collections for soursop (30 accessions), cherimoya (70 accessions) and Ilama (100 collections) only. The seed is not orthodox and conservation in the middle and long term is not yet possible in Mexico. Potential uses for this edible-fruit group of plants is high but growing problems as varietal diversity, plant protection, harvest technology and information of local markets limit the crop.
\end{abstract}

Index terms: Entobotany, uses, consevation, Annonaceae.

${ }^{1}$ Palestra Anonáceas - V Congresso Internacional \& Encontro Brasileiro sobre Annonaceae: do gene à exportação (19 a 23 de Agosto de 2013). Botucatu-SP.

${ }^{2}$ CRUCO-UACH. Av. Periférico Independencia Poniente No.1000. C.P. 58000. Morelia, Michoacán, México. Email: aajorge2000@ yahoo.com.mx; ssegura@correo.chapingo.mx 


\section{INTRODUCCIÓN}

La Angiosperm Phylogeny Group (1998) consigna que las anonáceas (Annonaceae) son una familia de Angiospermas del Orden Magnoliales, que consta de 130 géneros con unas 2300 especies que se distribuyen por los trópicos del Nuevo y Viejo Mundo, hasta el norte de Australia y las islas del Pacífico y forman parte del sistema ranaliano, lo que las hace tener una historia evolutiva muy antigua.

Chatrou (1999) indica que África es el continente que contiene el número más bajo de especies, aproximadamente 450, cerca de 900 se encuentran en los Neotrópicos, casi 1200 en las áreas tropicales de Asia y Australia. El mismo autor agrega que de todos los géneros solamente cuatro: Annona, Rollinia, Uvaria y Asimina producen frutos comestibles. La familia se concentra en los trópicos, con pocas especies que se encuentran en las regiones templadas (Asimina sp.) y el chirimoyo (A. cherimola Mill.) se localiza en los subtrópicos. Alrededor de 900 especies son Neotropicales, 450 son Afrotropicales, y las otras especies de la región Indomalaya.

Annonaceae tienen amplia diversidad de géneros y especies cuya distribución está localizada principalmente en el trópico y el chirimoyo en el subtrópico. La mayoría de las especies se encuentran localizadas en las áreas naturales y algunas cultivadas en huertos comerciales y de traspatio. En estos últimos sistemas de cultivo es donde se observa mucha diversidad propiciada por muchos años de cultivo, que actualmente por intervención humana irracional y los factores ambientales adversos derivados del cambio climático se encuentra con riesgos de erosión y pérdida. Por lo tanto, el uso sostenible depende del estudio de tal variación y de la conservación apropiada (ANDRÉS et al., 2006).

En México en el año 2002 se constituyó la Red Mexicana de Anonáceas (REMA), organización cuyo objetivo central es la gestión de los recursos genéticos de especies del género Annona y géneros silvestres relacionados, que ante la amenaza cada vez mayor del deterioro ambiental se encuentran en riesgo de erosión genética y pérdida gradual; la REMA asocia actualmente 7 profesionales de 5 instituciones de educación superior y centros de investigación del sector público para desarrollar investigaciones relacionadas con la prospección, caracterización, selección, conservación y uso de los recursos fitogenéticos con apoyos financieros del Sistema Nacional de Recursos Filogenéticos (SINAREFI) del sistema Nacional de Inspección y Certificación de Semillas (SNICS) de la Secretaria de Agricultura, Ganadería y Desarrollo Rural
(SAGARPA).

En estudios de diagnósticos realizados en 2010 hemos ubicado en México 14 géneros y 63 especies de la familia Annonaceae hasta ahora identificados y de éstas las de mayor importancia económica son: guanábana (A. muricata L.), chirimoya ( $A$. cherimola Mill.), saramuyo ( $A$. squamosa L.), ilama o papausa (A.diversifolia Saff.), anona colorada o amarilla ( $A$. reticulata L.) y chincuya o cabeza de negro (A. purpurea L.) (ANDRÉS-AGUSTÍN y ANDRÉS-HERNÁNDEZ, 2011) (Cuadro 1).

\section{La Conservación de recursos genéticos de Annonaceae en México}

De las siete especies frutales de Annona que se encuentran en México, salvo la guanábana, todas presentan una variabilidad que no se restringe en los tipos cultivados. Las comunidades humanas donde se distribuyen, las siguen domesticando (SEGURA y ANDRÉS,2011). La Figura 1 es una muestra de lo anterior. En seguida presentamos las principales características de la conservación en el país.

\section{Conservación ex situ}

Las semillas de Annona no son ortodoxas y su embrión no está maduro cuando se cosechan los frutos. Esto obliga a buscar métodos de conservación de semillas tales como la crioconservación (GEORGE and NISSEN, 1987). La variabilidad natural en poblaciones de saramuyo, ilama y chirimoya es un factor que dificulta la puesta en marcha de protocolos de germinación y conservación de semillas (GONZÁLEZ et al., 2005).

La conservación ex situ en México se está desarrollando por investigadores que pertenecen a la Red Mexicana de Anonáceas (REMA) en bancos de germoplasma de campo y semilla, principalmente. Los bancos y las accesiones que se conservan son principalmente selecciones de material sobresaliente en características agronómicas provenientes de los trabajos de caracterización y selección de material de traspatio y de huertos comerciales. Los bancos que actualmente se construyen están localizados principalmente en regiones tropicales, a continuación se indican en el Cuadro 3.

Por otro lado y con el objetivo de conservar germoplasma de A.cherimola Mill. en el Banco Nacional de Germoplasma Vegetal (BANGEV) localizado en el Departamento de Fitotecnia de la Universidad Autónoma Chapingo se realizaron recogidas de semillas de 39 accesiones y más de 11 mil semillas provenientes de los estados de Michoacán, Morelos, Oaxaca y Estado de México 
en los años 2010-2011. Posterior a la recogida el material se acondicionó para su conservación a largo plazo a un $15 \%$ de humedad relativa y a la temperatura promedio de $-20^{\circ} \mathrm{C}$.

\section{Conservación in situ}

Los objetivos de la conservación in situ son los de conservar la variabilidad genética (genes) de las especies de Annona de mayor importancia económica y reducir los riegos de erosión genética y pérdida de individuos. En las acciones de conservación de la REMA se hace énfasis en el fomento de la conservación mediante pláticas con los agricultores de la necesidad de la participación para conservar y utilizar los recursos genéticos de las anonas en sus huertos tradicionales (traspatio) y comerciales, así como de áreas naturales (conservación participativa) mediante acciones de uso racional. Estos riesgos surgen por la introducción de nuevos cultivos, por la construcción de caminos y vivienda. La gran riqueza genética de la mayoría de las especies de Annona, por fortuna, está siendo conservada por los agricultores debido al valor de uso comestible y medicinal. Aunque existen riesgos, este está siendo disminuido por la práctica de la agricultura tradicional y la recolección de las anonas por los agricultores rurales. La Red participa por lo tanto, con acciones de fomento en la conservación in situ y en algunos casos mediante la propagación de plantas para entregar a los productores las especies en que sus poblaciones se ven disminuidas, como en Yucatán con Annona squamosa. Las especies de mayor importancia económica como guanábana, chirimoya, saramuyo, anona amarilla, ilama y la chincuya se conservan en los huertos de traspatio y la gran mayoría de las especies silvestres en áreas naturales con vegetación natural.

La conservación in situ de Annona en México como en muchas otras especies se enfrenta a la disyuntiva del fomento del cultivo vía selección y por ende deriva genética. Segura et al. (2010) describen que comunidades indígenas de la región de Costa Chica de Guerrero, mantienen algunos tipos de ilama y la tendencia en esa región es de ir eliminando tipos no preferidos. El acondicionamiento de la pulpa se presenta como una opción para ampliar el tiempo de disponibilidad de pulpa de esta especie en la región.

\section{Usos de las anonas}

Las especies de Annona que figuran en las estadísticas de producción son: guanábana (Annona muricata L.), chirimoya (A.cherimola MIll.) y saramuyo (A.squamosa L.). La superficie cosechada de guanábana fue de 2,333 ha, la producción de
20,050 toneladas con el rendimiento promedio de 8.5 ton/ha. en los estados de Nayarit, Colima, Guerrero, Michoacán y Tabasco. En el caso de chirimoya se cosecharon 90 ha, se produjeron 609 toneladas con el rendimiento promedio de 6.77 ton/ha en los estados de Morelos, Michoacán e Hidalgo y finalmente para el caso de saramuyo la superficie cosechada fue de 63 ha, la producción de 544 toneladas y el rendimiento medio de 8.5 ton/ha exclusivamente en el estado de Yucatán. El resto de las especies de Annona no figuran en las estadísticas oficiales, lo que indica que su superficie es muy reducida o no se cultivan comercialmente pero existen en huertos de traspatio o en áreas naturales de donde se recolectan sus frutos para autoconsumo (ANDRÉS-AGUSTÍN,1997).

La guanábana es un frutal posiblemente originario de Suramérica, que tuvo una amplia expansión en tiempos prehispánicos; que no se conoce en estado silvestre y al contrario de las demás Anonáceas, A. muricata se consume poco como fruta fresca en México, pues se usa principalmente en la preparación de refrescos y helados, ya que la pulpa en la mayoría de las variedades es muy ácida y aromática (PINTO, 2005). Recientemente en el estado de Nayarit se ha fomentado su cultivo de manera significativa y existe una superficie cultivada de más de 2 mil hectáreas.

A pesar de los avances en el conocimiento de la ciencia etnobotánica en México, los estudios etnobotánicos específicos en Annonaceae son escasos. Farrera-Sarmiento (2011) realizó un estudio sobre los aspectos etnobotánicos de Annonaceae en Chiapas, México, orientado a conocer las especies de útiles Annonacea en el estado de Chiapas. El autor señala los principales usos de 20 especies agrupadas de 6 géneros para fines comestibles como fruta fresca y medicinales en sus distintas aplicaciones como en problemas gastrointestinales, respiratorios, fiebre, paludismo, dolor de cabeza, gripe, sinusitis, hemorragia nasal, golpes, fuego bucal, cruda, renal, vómito, principalmente de las especies del género Annona; de menor frecuencia encontró el uso artesanal, leña, construcción, forraje, sombra de cacaotales y cafetales y ornato. Estudios científicos más recientes de algunas especies de anonáceas para propósitos antibacteriales, antifúngicos y antihelmínticos son señalados por Padjama et al. (1993) y para usos insecticidas por Alvarez et al. (2007). Particularmente en México crece entre la población el uso de los extractos de la hoja de Annona muricata para fines antitumorales.

Por su parte Segura et al. (2011) condujo un estudio sobre el uso y la conservación de ilama (Annona diversifolia Saff.) en 3 comunidades de 
Tierra Caliente en el estado de Guerrero, México y concluye que la ilama tiene un gran impacto económico para la gente que se dedica a la recolección de frutos de áreas naturales o semicultivada en los pequeños huertos de traspatio, pero puede traer consecuencias negativas al incrementar su cultivo ya que puede desplazar al sector recolector en pequeña escala si se concentra la producción en unos cuantos agricultores. Agrega el mismo autor que el manejo de la ilama varía entre comunidades.

En el diagnóstico se encontró que los usos principales de las especies de Anona son: pulpa comestible de los frutos en forma fresca o congelada (guanábana), elaboración de licores, helados; usos medicinales para aliviar diversas enfermedades; leña o combustible, postes para cercos o sombra del cafeto. Lo anterior coincide con lo señalado por Farrera-Sarmiento (2011). Estudios para dar un mejor aprovechamiento de guanábana fueron realizados por Alpuche et al. (2006) quienes concluyen que es posible dar el uso más integral de la cáscara para compostas, las semillas para la extracción de acetogeninas como insecticidas, el aceite por su alta viscosidad para la lubricación de motores de dos tiempos como motocicletas y la pulpa por su corta vida de anaquel recomienda utilizarla en forma congelada para helados, refrescos y jarabes.

Finalmente es necesario señalar que la Sociedad Mexicana de Anonáceas (SOCMEXAN) y en particular la Red Mexicana de Anonáceas (REMA) del Sistema Nacional de Recursos Genéticos (SINAREFI) son las organizaciones de profesionales que han impulsado los estudios de Conservación y Utilización de este grupo de frutales en México en años recientes.

CUADRO 1- Familia Annonaceae, Géneros y especies en México.

\begin{tabular}{|c|c|c|}
\hline Género & Especies & Distribución (Entidades Federativas) \\
\hline 1.Anaxagorea & 1.A. guatemalensis & Ver., Oax. \\
\hline 2.Annona & $\begin{array}{l}\text { 2.A. globiflora } \\
\text { 3.A. testudinea } \\
\text { 4.A. cherimola } \\
\text { 5.A. longiflora } \\
\text { 6.A. macrophyllata } \\
\text { 7.A. primigenia } \\
\text { 8.A. reticulata } \\
\text { 9.A. glabra } \\
\text { 10.A. purpurea } \\
\text { 11.A. diversifolia } \\
\text { 12.A. lutescens } \\
\text { 13. A. longipes } \\
\text { 14. A. scleroderma } \\
\text { 15.A. muricata } \\
\text { 16.A. squamosa }\end{array}$ & $\begin{array}{l}\text { Ver., Hgo., Chis., Tamps., Gto. } \\
\text { Chis. } \\
\text { Ver.,Mich.,Chis.,Oax.,Mor.,Pue.,Qro.,Jal.,Gto.,M } \\
\text { éx. } \\
\text { Jal.,Ver. } \\
\text { Ver., Camp. } \\
\text { Camp.,Q.Roo.,Yuc. } \\
\text { Yuc.,Jal.,Mich.,Pue.,Q.Roo,Nay.,Tab. } \\
\text { Ver.,Q.Roo,Yuc., Camp.,Tab.,Jal.,Oax.,Nay.,Gro. } \\
\text { Ver.,Mich.,Chis.,Yuc.,Jal. } \\
\text { Mich.,Gro.,Yuc.,Ver.,Méx. } \\
\text { Pue. } \\
\text { Ver. } \\
\text { Chis. } \\
\text { Ver.,Chis.,Tab.,Yuc.,Col.,Gro.,Q.Roo, Nay.,Mich. } \\
\text { Yuc.,Mich.Q.Roo,Camp.,Ver.,Oax., Jal.,Nay. }\end{array}$ \\
\hline 3.Desmopsis & $\begin{array}{l}\text { 17.D. trunciflora } \\
\text { 18. D. lanceolata } \\
\text { 19.D. trunciflora } \\
\text { 20.D. trunciflora var. glabra } \\
\text { 21.D.galeottiana } \\
\text { 22.D.uxpanapensis } \\
\text { 23.D. mexicana } \\
\text { 24.D. schippii } \\
\text { 25.D.bibracteata }\end{array}$ & $\begin{array}{l}\text { Jal. } \\
\text { Chis. } \\
\text { Ver.,Oax.,Chis. } \\
\text { Ver. } \\
\text { Ver,Jal.,Gro.,Chis. } \\
\text { Chis.,Ver. } \\
\text { Mich.(Aquila, Coalcoman) } \\
\text { Tab. } \\
\text { Ver. }\end{array}$ \\
\hline
\end{tabular}




\begin{tabular}{|c|c|c|}
\hline 4.Cymbopetalum & $\begin{array}{l}\text { 26.C. hintonii } \\
\text { 27.C. mayanum } \\
\text { 28.C.galeottiana } \\
\text { 29.C.penduliflorum } \\
\text { 30.C.baillonii }\end{array}$ & $\begin{array}{l}\text { Mich.(Aquila) } \\
\text { Chis. } \\
\text { Ver.,Jal.,Chis. } \\
\text { Ver.,Chis } \\
\text { Ver. }\end{array}$ \\
\hline 5.Guamia & 31. G. mexicana & Ver. \\
\hline 6.Guatteria & $\begin{array}{l}\text { 32.G. anómala R.F.Fr. } \\
\text { 33.G.amplifolia } \\
\text { 34.G.depressa } \\
\text { 35.G.dolichopoda } \\
\text { 36.G.bibracteata } \\
\text { 37.G.diospyroide } \\
\text { 38.G.grandiflora } \\
\text { 39. G.oliviformis } \\
\text { 40.G.galeottiana } \\
\text { 41.Guatteria } \mathrm{sp} \text {. }\end{array}$ & $\begin{array}{l}\text { Chis.,Ver. } \\
\text { Chis.,Ver.,Tab.,Jal. } \\
\text { Ver.,Chis. } \\
\text { Ver. } \\
\text { Ver. } \\
\text { Ver.,Oax. } \\
\text { Chis. } \\
\text { Chis. } \\
\text { Ver., Oax. } \\
\text { Ver. }\end{array}$ \\
\hline 7.Malmea & $\begin{array}{l}\text { 42.M. depressa } \\
\text { 43. M. gaumeri }\end{array}$ & $\begin{array}{l}\text { Chis., Ver., Camp.,Mich.,Tab., Oax.,Q.Roo,Yuc. } \\
\text { Ver.,Chis. }\end{array}$ \\
\hline 8.Oxandra & $\begin{array}{l}\text { 44.O. lanceolata } \\
\text { 45. O.lanceolata spp. } \\
\text { macrocarpa } \\
\text { 46.O. laurifolia } \\
\text { 47.O. maya Miranda }\end{array}$ & $\begin{array}{l}\text { Mich.,Gro.,Jal.,Q.Roo } \\
\text { Gro.(José Azueta) } \\
\text { Chiapas }\end{array}$ \\
\hline 9.Tridimeris & $\begin{array}{l}\text { 48.T. hahniana } \\
\text { 49.T.tuxtlensis } \\
\text { 50. T.uxpanapensis }\end{array}$ & $\begin{array}{l}\text { Ver. } \\
\text { Ver. } \\
\text { Oax. }\end{array}$ \\
\hline 10.Rollinea & $\begin{array}{l}\text { 51.R. jimenezii } \\
\text { 52.R.mucosa } \\
\text { 53.R.rensoniana }\end{array}$ & $\begin{array}{l}\text { Ver., Chis. } \\
\text { Ver. } \\
\text { Ver.,Oax. }\end{array}$ \\
\hline 11.Sapranthus & $\begin{array}{l}\text { 54.S. humilis } \\
\text { 55.S. campechianus } \\
\text { 56.S. foetidus } \\
\text { 57.60. S.microcarpus } \\
\text { 58.Sapranthus sp. }\end{array}$ & $\begin{array}{l}\text { Ver. } \\
\text { Yuc.,Camp.,Q.Roo } \\
\text { Jal. } \\
\text { Ver., Chis., Jal., Oax. } \\
\text { Nay.,Oax. }\end{array}$ \\
\hline 12.Xylopia & $\begin{array}{l}\text { 59.X. sericophylla } \\
\text { 60.X. frutescens }\end{array}$ & $\begin{array}{l}\text { Ver. } \\
\text { Tab.,Chis.,Ver.,Oax. }\end{array}$ \\
\hline 13.Mosannona & $\begin{array}{l}\text { 61.M. depressa } \\
\text { 62. M.depressa spp. depressa }\end{array}$ & $\begin{array}{l}\text { Ver. } \\
\text { Ver., Q.Roo }\end{array}$ \\
\hline 14.Stenannona & 63. S. humilis & Tab., Ver. \\
\hline
\end{tabular}

Fuente: Trabajo de campo y herbarios de la REMIB.(Red Mundial de Información sobe Biodiversidad). 
CUADRO 2- Principales especies frutales de Annona en México.

\begin{tabular}{|c|c|c|c|}
\hline Especies & Nombre común & Altitud (m) & Distribución en Méx ico \\
\hline A. cherimola M ill. & Chirimoya & $700-2600$ & Chis, Gto, Gro, Hgo, Mex, Mich, Mor, Jal, Oax, Pue, Ver, Yuc \\
\hline A. muricata $\mathrm{L}$. & Guanabana & $0-500$ & Col., Sin., Nay., M ich., Jal., Ver., Oax., Chis., Tab., Yuc. \\
\hline A. squamosa L. & Saramuyo & $0-1000$ & Chis., Camp., Yuc., Q.R oo \\
\hline A. purpurea & Chincuya & $0-1200$ & Col., Sin., Nay., M ich., Jal., Ver., Oax., Chis., Tab, Camp., Yuc. \\
\hline A. reticulata $\mathrm{L}$. & Anona colorada & $50-1250$ & Ver., Tab., Chis., Mich., Oax., Yuc. \\
\hline A. globiflora Sch. & Anona de monte & $0-1000$ & Tamps., SLP., Ver., Hgo. \\
\hline A. diversifolia Saff. & llama & $250-600$ & Col., Gro., Mich., M ex., Oax., Chis. \\
\hline
\end{tabular}

CUADRO 3 - Conservación ex situ (bancos de campo) de especies de Annona en México por la Red de Annonaceae.

\begin{tabular}{llc}
\hline \multicolumn{1}{c}{ Especie } & \multicolumn{1}{c}{ Ubicación del banco/Institución } & No.de accesiones en el banco $^{\circ}$ \\
\hline Annona muricata & Actopan,Ver-Universidad Veracruzana & 30 \\
Annona muricata & Santiago Ixcuintla,Nay.-INIFAP* & 5 \\
Annona squamosa & Uxmal,Yuc.-INIFAP & 30 \\
Annona diversifolia & $\begin{array}{l}\text { Cd. Altamirano,Gro.-Instituto Tecnológico de } \\
\text { Ciudad Altamirano }\end{array}$ & 100 \\
Annona cherimola & $\begin{array}{l}\text { Coatepec Harinas, México-Fundación } \\
\text { Salvador Sánchez Colín (CICTAMEX, S.C.) }\end{array}$ & 70 \\
Annona reticulata & Uxmal,Yuc.-INIFAP & 30 \\
Otras especies & Uxmal,Yuc.-INIFAP & 30 \\
\hline *. Institur Nacio & & \\
\hline
\end{tabular}

*: Instituto Nacional de Investigaciones Agropecuarias y Forestales.

\section{CONCLUSIONES}

1-De las 63 especies de Anonáceas que existen en México, siete son aprovechadas como frutales y solamente la Guanábana, Chirimoya, Ilama y Saramuyo son comercializadas. Los mercados locales son los principales lugares donde se compran los frutos.

2-Se estima que la conservación in situ es el método más generalizado para mantener las especies de Annona en México y en este tipo de conservación el medio natural pero sobretodo las comunidades humanas son factores determinantes. La conservación ex situ comprende siete bancos in vivo de la REMA que guardan 295 accesiones. Los protocolos para la conservación a mediano o largo plazo no existen pues se trata de especies cuya semilla no es ortodoxa. La crioconservación pudiera ser la vía a seguir para este propósito.

3- La Sociedad Mexicana de Anonáceas (SOCMEXAN) y en particular la Red Mexicana de Anonáceas (REMA) del Sistema Nacional de Recursos Genéticos (SINAREFI) son las organizaciones de profesionales que han impulsado los estudios de Conservación y Utilización de este grupo de frutales en México en años recientes.

4-De acuerdo a las estadísticas nacionales, las especies de Annona que son cultivadas en forma comercial son: guanábana cuya producción es de 20,050 ton., la chirimoya con 609 ton. y saramuyo con 544 ton.

5- El potencial de cultivo de las anonas es alto en México por contar con grandes áreas tropicales y subtropicales. Las principales limitantes son: la falta de calidad de frutos y la pulpa, reducidos mercados, daños por plagas y enfermedades y la falta de técnicas apropiadas de cultivo. Los estudios etnobotánicos indican que los principales usos de la especies del género Annona son principalmente para fines alimenticios de la pulpa de la fruta ya sea en forma fresca o congelada, elaboración de cremas pastas y licores y secundariamente para propósitos medicinales para cura a muchas enfermedades. 


\section{AGRADECIMIENTOS}

Este trabajo está dedicado a la Profa. Lila Marroquín Andrade por su impulso a la conformación de la SOCMEXAN y la REMA. La UACh y el SINAREFI otorgaron los fondos del proyecto REMA 2012.

\section{REFERENCIAS}

ALPUCHE-SILVESTRE, E.; GARCÍA-AGUILAR, L.; BOLIVAR FERNÁNDEZ, N. Aprovechamiento integral de la guanábana (Annona muricata L.). In: CONGRESO NACIONAL DE Annonaceae, 3., 2006., Villahermosa. Memoria... p.58

ALVAREZ, C. O.; NESKE, A. ; POPICH, S.; BARDON, A. Toxic effects of annonaceous acetogenins from Annona cherimola on Spodoptera frugiperda (Lepidoptera: Noctuidae). Journal of Pest Science, Heidelberg, v.80, p.63-67, 2007.

ANDRES-AGUSTIN, J. El cultivo de chirimoya (Annona cherimola Mill.) en el estado de Michoacán, México. Proceedings of Interamerican Society for Tropical Horticulture, Guatemala, v.41, p.152-161, 1997

ANDRÉS-AGUSTÍN, J.; ANDRÉS-HERNÁNDEZ, L. Biología, diversidad, conservación y uso sostenible de los recursos genéticos de Annonaceae en México. Chapingo: Universidad Autónoma Chapingo, 2011. 141 pp.

ANDRES-AGUSTIN, J.; GONZALEZ-ANDRES, F.; NIETO-ANGEL, R.; BARRIENTOS-PRIEGO, A.F. Morphometry of the organs of cherimoya (Annona Cherimola Mill.) and analysis of fruit parameters for the characterization of cultivars, and Mexican germoplasm selections. Scientia Horticulturae, Amsterdam, v.107, p. 337-346, 2006.

ANGIOSPERM PHYLOGENY GROUP. An ordinal classification for the families of flowering plants. Annals of the Missouri Botanical Garden, Saint Louis, v.85, p.531-553, 1998.

CHATROU, L. W. The Annonaceae and the Annonaceae Project: a brief overview of the state of affairs. Acta Horticulturae, The Hague, n.497, p. 43-49, 1999.
FARRERA-SARMIENTO, O. Aspectos etnobotánicas de anonáceas en Chipas, México. In: GONZÁLEZ-ESQUINCA, A.R.; MERCEDESCAZARES, L.; GUTIÉRREZ-JIMÉNEZ, J.; SCHLIE-GUZMÁN, M.A.; VIDAL-LÓPEZ , D.G. (Coord.). Anonaceas: plantas antiguas, estudios recientes. Tuxtla: Universidad de Ciencias y Artes de Chiapas, 2011. p.121-128.

GEORGE, A.P.; NISSEN, R. J. Propagation of Annona species. Scientia Horticulturae, Amsterdam, v.33, p.75-85, 1987.

GONZÁLEZ G. J.; GUZMÁN G.S.; MICHEL R.A.; RADILLO, J.R.; BAZAN T. M.; CERVANTES T.F. Evaluation of pregerminative treatmentsin Soursop (Annona muricata L.) Seeds HortScience, Alexandria, v.40, p.1023, 2005.

GONZÁLEZ G.J.M.; RADILLO, J.F.; BAZAN T.M.; GONZÁLEZ R.J.C. Evaluation of three grafting methods for propagating soursop (Annona muricata L.) in the dry tropic region of Mexico. HortScience, Alenxadria, v.40, p.1082, 2005.

PADJAMA, V.; THANKAMANY, V.; HISHAM, A. Antibacterial, antifungal and antihelmintic activities of root barks of Uvaria hooki and Uvaria narum. Journal of Etnopharmacology, Lausanne, v.40, p.181-186, 2005.

PINTO, A. C. DE Q.; CORDEIRO, M.C.R.; ANDRADE, S. R. M. DE; FERREIRA, F. R.; FILGUEIRAS, H. A. DE C.; ALVES, R. E.; KINPARA, D. I. Annona species. Southampton: International Centre for Underutilised Crops, 2005.

SEGURA, L.S.; ANDRES, J. Where do we go with Annona in Mexico?. In: INTERNATIONAL SYMPOSIUM ON WILD RELATIVES OF SUBTROPICAL AND TEMPERATE FRUIT AND NUT CROPS, 1., 2011. Davis. Proceedings...

SEGURA, S.; LEFEVRE, C.; CARMONA, A.; MARROQUÍN, L. Underutilization of the genetic resources of Annona diversifolia Saff. in the communities of Tierra Caliente, México. In: SIMPOSIO INTERNACIONAL DE RECURSOS GENÉTICOS DE AMÉRICA LATINA Y EL CARIBE, 8., 2011, Quito. Proceedings... 